\title{
ON EMBEDDINGS AND TRACES IN SOBOLEV SPACES WITH WEIGHTS OF POWER TYPE
}

\author{
JIR̃í RÁKOSNÍK \\ Matematický Ústar CSAV, Žitná 25, 11567 Praha 1, Czechoslovakia
}

\section{Introduction}

Let $\boldsymbol{R}^{N}$ be the $N$-dimensional Euclidean space, let $\Omega \subset \boldsymbol{R}^{N}$ be a bounded (open) domain and let $M$ be a non-empty closed subset of $R^{N}-\Omega$. For $x \in R^{N}$ set

$$
d_{M}(x)=\operatorname{dist}(x, M) \text {. }
$$

Let $\varepsilon \in R, k \in N, 1<p<\infty$. The weighted Sobolev space $W^{k, p}\left(\Omega ; d_{M}, \varepsilon\right)$ is the set of all measurable functions $u$ on $\Omega$ such that

$$
\|u\|_{W}=\left(\sum_{|\alpha| \leqslant k} \int_{\Omega}\left|D^{\alpha} u(x)\right|^{p} d_{M}^{\varepsilon}(x) d x\right)^{1 / p}<\infty
$$

where $\alpha=\left(\alpha_{1}, \ldots, \alpha_{N}\right) \in N_{0}^{N},|\alpha|=\alpha_{1}+\ldots+\alpha_{N}$ and the $D^{\alpha} u$ are distributional derivatives of $u$. The expression (1) defines a norm in $W^{k, p}\left(\Omega ; d_{M}, \varepsilon\right)$ which provided with this norm is a Banach space.

In [5] and [1] it was proved that under certain assumptions on $\varepsilon, k, p$, $\Omega$ and $M$ the space $W^{k, p}\left(\Omega ; d_{M}, \varepsilon\right)$ is continuously embedded in another weighted space of Sobolev type $H^{k, p}\left(\Omega ; d_{M}, \varepsilon\right)$ which consists of all functions $u$ such that

$$
\|u\|_{H}=\left(\sum_{|x| \leqslant k} \int_{S \Omega}\left|D^{\alpha} u(x)\right|^{p} d_{M}^{\varepsilon-(k-|\alpha|) p}(x) d x\right)^{1 / p}<x .
$$

$\left(H^{k, p}\left(\Omega ; d_{M}, \varepsilon\right)\right.$ is also a Banach space when equipped with the norm (2).)

The spaces $H^{k, p}\left(\Omega ; d_{M}, \varepsilon\right)$ are worth studying for several reasons: the exponents of the weight $d_{M}$ in (2) are less than the ones in (1), so that the norm (2) reflects more finely the behaviour of functions; in (2) there may be exponents of different signs, i.e. the norm (2) admits simultaneous appearance of weights with both degeneracy and singularity; the spaces $H^{k, p}\left(\Omega ; d_{M}, \varepsilon\right)$ occur in applications to boundary value problems. 
Let $W_{M}^{k, p}\left(\Omega ; d_{M}, \varepsilon\right)$ and $H_{M}^{k, p}\left(\Omega ; d_{M}, \varepsilon\right)$ be the closures of the set

$$
C_{M}^{\infty}(\bar{\Omega})=\left\{v \in C^{\infty}(\bar{\Omega}) ; \operatorname{supp} v \cap M=\emptyset !\right.
$$

in the spaces $W^{k, p}\left(\Omega ; d_{M}, \varepsilon\right)$ and $H^{k, p}\left(\Omega ; d_{M}, \varepsilon\right)$ respectively. In [1] it is claimed that for all $\varepsilon \in \boldsymbol{R}$,

$$
H^{k, p}\left(\Omega ; d_{M}, \varepsilon\right) \subset W_{M}^{k, p}\left(\Omega ; d_{M}, \varepsilon\right)
$$

(the symbol $\leftrightharpoons$ denotes continuous embedding). Unfortunately, that assertion (Theorem 1.2 in [1]) does not hold, because the assumptions on $\Omega$ and $M$ are too weak and in the proof the estimate preceding (1.13) contains a mistake. We shall give here a correct version of the theorem proving at the same time a bit more: the embedding into $H_{M}^{k, p}\left(\Omega ; d_{M}, \varepsilon\right)$. In Section 3 we shall discuss the existence and value of traces of functions from weighted Sobolev spaces.

\section{Embeddings}

Throughout this section we shall suppose that the domain $\Omega$ has the segment property outside the set $M$ and satisfies the inner cone condition in a neighbourhood of the boundary of the set $\partial \Omega-M$; more precisely: There exists an open covering $\left\{U_{j}\right\}_{j=1}^{s}$ of $\bar{\Omega}$ with the following properties:

(a) If

$$
\bar{U}_{j} \cap M=\emptyset,
$$

then there exists a vector $\xi_{j} \in R^{N}-\{0\}$ such that $x+t \xi_{j} \in \Omega$ for all $x \in U_{j} \cap \bar{\Omega}$ and $0<t<1$.

(b) If

$$
U_{j} \cap M \cap \overline{\partial \Omega-M} \neq \varnothing,
$$

then there exists an open cone $C_{j}$ with vertex at the origin, congruent to a given cone $C$, and such that $\left(x+C_{j}\right) \subset \Omega$ for all $x \in U_{j} \cap \bar{\Omega}$.

(c) If neither (3) nor (4) holds then $U_{j} \cap(\partial \Omega-M)=\emptyset$.

Theorem 1. Let $1<p<\infty, k \in N$ and $\varepsilon \in R$. Then

$$
H^{k, p}\left(\Omega ; d_{M}, \varepsilon\right)=H_{M}^{k, p}\left(\Omega ; d_{M}, \varepsilon\right) .
$$

Proof. Let $u \in H^{k, p}\left(\Omega ; d_{M}, \varepsilon\right)$. Evidently, it suffices to find a sequence of functions $w_{n} \in C_{M}^{\infty}(\bar{\Omega})$ converging to $u$ in $H^{k, p}\left(\Omega ; d_{M}, \varepsilon\right)$. For $h>0, x \in R^{N}$, denote by $B_{h}(x)$ the ball in $R^{N}$ of radius $h$ with center at $x$. Let.$_{i}^{\prime} \psi_{j}^{\prime \prime}{ }_{j=1}^{\prime s}$ be a partition of unity on $\bar{\Omega}$ subordinate to the covering $\left\{U_{j}\right\}_{j=1}^{s}$. Put $u_{j}=u \psi_{j}$ and extend it by zero outside $\Omega$. Let $\varphi \in C_{0}^{\alpha}\left(R^{N}\right)$ be a non-negative function such that $\operatorname{supp} \varphi \subset B_{1}(0), \int_{a^{N}} \varphi(x) d x=1$ and put $\varphi_{h}(x)=h^{-N} \varphi(x / h)$ for 
$h>0$. Write $\delta=\min \operatorname{dist}\left(U_{j}, M\right)$, where the minimum is taken over all $j$ satisfying (3). For a function $v$ on $\Omega$ and $t>0$ define $v^{(t)}(x)=v(x)$ if $d_{M}(x)$ $\geqslant t$ and $x \in \Omega, v^{(t)}(x)=0$ otherwise.

Take $j=1, \ldots, s$.

First suppose that (3) holds. Then $\delta \leqslant d_{M}(x) \leqslant \operatorname{diam} \Omega+\operatorname{dist}(\Omega, M)$ $<\infty$ for $x \in U_{j} \cap \Omega$, so that $H^{k, p}\left(U_{j} \cap \Omega ; d_{M}, \varepsilon\right)$ coincides with the nonweighted Sobolev space $W^{k, p}\left(U_{j} \cap \Omega\right)$ and we can construct in a usual way, by the use of translation and mollification arguments, a sequence of functions $v_{j, h} \in C^{\infty}\left(R^{N}\right)$ such that

$$
\begin{gathered}
\operatorname{supp} v_{j, k} \cap M=\varnothing, \\
v_{j, h} \rightarrow u_{j} \text { in } H^{k, p}\left(\Omega ; d_{M}, \varepsilon\right) \quad \text { as } h \rightarrow 0
\end{gathered}
$$

Next, suppose (4). The cone $C_{j}$ from condition (b) can be expressed in the form

$$
C_{j}=\bigcup_{0<i<1} t B_{r}\left(\xi_{j}\right)
$$

where $\xi_{j} \in R^{N}-\{0\}, 0<r<\left|\xi_{j}\right|$. Set $\sigma=r^{-1}\left|\xi_{j}\right|>1$ and define functions $v_{j, h} \in C^{\infty}\left(R^{N}\right), h>0$, by

$$
v_{j, h}(x)=\int_{\tilde{N}^{N}} \varphi_{h}(x-y) u_{j}^{(5 \sigma h)}\left(y+h r^{-1} \xi_{j}\right) d y .
$$

We shall prove that the $v_{j, h}$ satisfy (5) and (6). The index $j$ will be omitted.

(i) If $x \in U \cap \Omega$ is such that $d_{M}(x) \leqslant 3 \sigma h$, then for $y \in B_{h}(x)$ we have $d_{M}\left(y+h r^{-1} \xi\right) \leqslant d_{M}(x)+|y-x|+h r^{-1}|\xi|<5 \sigma h$. Hence,

$$
v_{h}(x)=0
$$

and (5) holds.

(ii) Let $x \in U \cap \Omega$ be such that $3 \sigma h<d_{M}(x) \leqslant 7 \sigma h$. Then for $y \in B_{(1+\sigma) h}(x)$ we get $\sigma h<d_{M}(y)<9 \sigma h$, i.e. $d_{M}(x) \sim d_{M}(y) \sim h(a \sim b$ means that the ratio $a / b$ is bounded from above and from below by positive constants). Hence, if $|\alpha| \leqslant k$, we can write

$$
D^{\alpha} v_{h}(x)=h^{-N-|\alpha|} \int_{\hat{\alpha}^{N}}\left(D^{\alpha} \varphi\right)\left(\frac{x-y}{h}\right) u^{(5 \sigma h)}\left(y+h r^{-1} \xi\right) d y
$$

and

$$
\begin{aligned}
\left|D^{\alpha} v_{h}(x)\right| & \leqslant \sup _{z}\left|D^{\alpha} \varphi(z)\right| h^{-N-|\alpha|} \int_{B_{h}(x)}\left|u\left(y+h r^{-1} \xi\right)\right| d y \\
& \leqslant c_{1} h^{-N-|\alpha|} \int_{\left.B_{(1}+\sigma\right) h(x)}|u(y)| d y .
\end{aligned}
$$


It follows that

$$
\begin{aligned}
\left|D^{\alpha} v_{h}(x)\right| d_{M}^{\varepsilon / p^{-k+|\alpha|}}(x) & \leqslant c_{2}\left|B_{(1+\sigma) h}(x)\right|^{-1} \int_{B_{(1+\sigma) h}(x)}|u(y)| d_{M}^{\varepsilon / p-k}(y) d y \\
& \leqslant c_{2} M\left(u d_{M}^{\varepsilon / p^{-k}}\right)(x),
\end{aligned}
$$

where the $c_{i}$ are positive constants, $|B|$ denotes the Lebesgue measure of $B$ and $M$ is the Hardy-Littlewood maximal operator defined by

$$
M f(x)=\sup _{t>0}\left|B_{t}(x)\right|^{-1} \int_{B_{t}(x)}|f(y)| d y .
$$

(iii) Suppose that $x \in U \cap \Omega$ is such that for some $l \geqslant 7$, loh $<d_{M}(x)$ $\leqslant(l+1) \sigma h$. Then $y \in B_{(1+\sigma) h}(x)$ implies $5 \sigma h \leqslant(l-2) \sigma h \leqslant d_{M}(y) \leqslant(l+3) \sigma h$, i.e. $d_{M}(x) \sim d_{M}(y) \sim l h$, and $u^{(5 \sigma h)}(y)=u(y)$. Moreover, $x+C . \subset \Omega$ by (b), which together with (7) yields $B=B_{h}\left(x+h r^{-1} \xi\right) \subset \Omega$. Thus for $|\alpha| \leqslant k$ we have

$$
\begin{aligned}
D^{\alpha} v_{h}(x) & =D^{\alpha} \int_{B} \varphi_{h}\left(x+h r^{-1} \xi-y\right) u(y) d y \\
& =h^{-N} \int_{B} \varphi_{h}\left(\frac{x-y}{h}+r^{-1} \xi\right) D^{\alpha} u(y) d y,
\end{aligned}
$$

and

(11)

$$
\begin{aligned}
\left|D^{\alpha} v_{h}(x)\right| d_{M}^{\varepsilon / p-k+|\alpha|}(x) & \leqslant \sup _{z}|\varphi(z)| h^{-N} \int_{B_{(1+\sigma) h}(x)}\left|D^{\alpha} u(y)\right| d_{M}^{\varepsilon / p-k+|\alpha|}(y) d y \\
& \leqslant c_{3} M\left(D^{\alpha} u d_{M}^{\varepsilon / p-k+|\alpha|}\right)(x)
\end{aligned}
$$

Put

$$
G(x)=\max _{|\alpha| \leqslant k} M\left(D^{\alpha} u d_{M}^{e / p-k+|\alpha|}\right)(x) .
$$

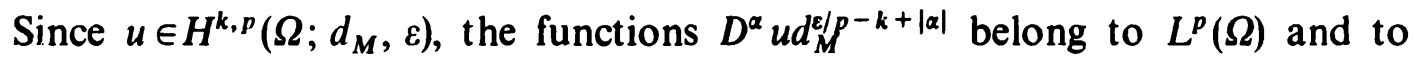
$L^{p}\left(R^{N}\right)$ as well. The boundedness of the maximal operator $M$ in $L^{p}\left(R^{N}\right)$ now implies that $G \in L^{p}\left(R^{N}\right)$ and by (9), (10), (11) we have

$$
\left|D^{\alpha} v_{h}(x)\right| d_{M}^{\varepsilon / p-k+|\alpha|}(x) \leqslant c G(x), \quad|\alpha| \leqslant k .
$$

It suffices to select a subsequence $\left\{v_{h_{n}}\right\}_{n=1}^{\infty}$ of $\left\{v_{h}\right\}_{h>0}$ such that

$$
D^{\alpha} v_{h_{n}}(x) \rightarrow D^{\alpha} u(x) \quad \text { for }|\alpha| \leqslant k \text { and for a.e. } x \in \Omega \text {. }
$$

Then by the Lebesgue Dominated Convergence Theorem, (6) holds with $h_{n}$ instead of $h$. The construction of $\left\{v_{h_{n}}\right\}$ relies on the properties of the mollifier and can be done in the same way as in the proof of Theorem 1.2 in [1].

Finally, if neither (3) nor (4) holds, then $(\partial \Omega-M) \cap U_{j}=\emptyset$. We define

$$
v_{j, h}(x)=\int_{\mathbb{R}^{N}} \varphi_{h}(x-y) u_{j}^{(3 h)}(y) d y
$$


and proceed like in the case of (4): if $d_{M}(x) \leqslant 2 h, 2 h<d_{M}(x) \leqslant 4 h$ or $l h<d_{M}(x) \leqslant(l+1) h, l \geqslant 4$, then (9), (10) or (11) holds respectively, and we again construct a subsequence of $\left\{v_{h}\right\}$ converging to $u$ in $H^{k, p}\left(\Omega ; d_{M}, \varepsilon\right)$.

The functions $w_{n}=\sum_{j=1}^{s} v_{j, h_{n}}$ form the desired sequence.

COROI.IARY. Under the assumptions of Theorem 1 the emhedding

$$
H^{k, p}\left(\Omega ; d_{M}, \varepsilon\right) \hookrightarrow W_{M}^{k, p}\left(\Omega ; d_{M}, \varepsilon\right)
$$

holds.

Proof. Since $\Omega$ is bounded, $d_{M}(x)<\operatorname{diam} \Omega+\operatorname{dist}(\Omega, M)<\infty$ and $\|u\|_{W} \leqslant c\|u\|_{H}$.

Remark 1. In other words, Theorem 1 and the corollary establish the density of the set $C_{M}^{\infty}(\bar{\Omega})$ in $H^{k, p}\left(\Omega ; d_{M}, \varepsilon\right)$ (with respect to both norms (1) and (2)).

We got the approximation by functions smooth up to the boundary $\partial \Omega$ at the cost of relatively strong assumptions. In [7] $\mathrm{H}$. Triebel proved (without any assumptions on $M$ and $\Omega$ ) that in the weighted (fractional order) Sobolev space $W^{s, p}\left(\Omega ; d_{M}, \varepsilon\right)$ the set $\left\{f ; f \in W^{s, p}\left(\Omega ; d_{M}, \varepsilon\right)\right.$, supp $f \cap M=\emptyset$; is dense.

Let us recall the inverse embedding proved in [1].

Proposition ([1], Theorem 2.3). Let $1<p<\infty, k \in N$ and $\varepsilon \in R$. Let $m \in\{0,1, \ldots, N-1\}, M \subset \partial \Omega$. Suppose that there exists an open covering $\left\{U_{i}\right\}_{i=0}^{\omega}(\omega \leqslant \infty)$ of $\bar{\Omega}$ with the properties:

(i) $\bigcup_{i=1}^{\infty)} U_{i} \supset M$, and there exists $s \in N$ such that every system of $s+1$ sets $U_{i}$ is disjoint;

(ii) $\bar{U}_{0} \cap M=\emptyset$;

(iii) there are numbers $c_{1}, c_{2}>0$ and a system of one-to-one mappings $T_{i}: \bar{Q} \rightarrow \overline{\Omega \cap U_{i}}, Q=(0,1)^{N}$, such that

$$
T_{i}\left(\left\{x \in \bar{Q} ; x_{m+1}=\ldots=x_{N}=0\right\}\right)=M \cap \bar{U}_{i}
$$

and

$$
c_{1}|x-y| \leqslant\left|T_{i}(x)-T_{i}(y)\right| \leqslant c_{2}|x-y| \quad \text { for all } x, y \in \bar{Q}, i=1,2, \ldots, \omega .
$$

Then

$$
V \hookrightarrow H^{k, p}\left(\Omega ; d_{M}, \varepsilon\right),
$$

where

$$
V=W^{k, p}\left(\Omega ; d_{M}, \varepsilon\right) \quad \text { if } \varepsilon>k p+m-N \text { or } \varepsilon \leqslant m-N \text {, }
$$

and

$$
V=W_{M}^{k, p}\left(\Omega ; d_{M}, \varepsilon\right) \quad \text { if } \varepsilon \neq j p+m-N, j=1, \ldots, k
$$


The absence of the values $\varepsilon=j p+m-N, j=1, \ldots, k$ in Proposition is essential. J. Kadlec and A. Kufner [2] proved for $\boldsymbol{\Omega}$ with Lipschitzian boundary and for $M=\partial \Omega$ that if $\varepsilon=j p-1$ with some $j=1, \ldots, k$, then $W_{0}^{k, p}\left(\Omega ; d_{M}, \varepsilon\right)\left(=W_{\partial \Omega}^{k, p}\left(\Omega ; d_{M}, \varepsilon\right)\right)$ is equivalent to the space $H_{G)}^{k, p}\left(\Omega ; d_{M}, \varepsilon\right)$ of functions with the norm

$$
\begin{aligned}
\|u\|_{H_{(j)}}=\left(\sum_{|x| \leqslant k-j} \int_{\Omega}\left|D^{\alpha} u(x)\right|^{p} d_{M}^{z-(k-|\alpha|) p}\left(\log \frac{R}{d_{M}(x)}\right)^{-p} d x\right. & \\
& \left.+\sum_{k-j<|\alpha| \leqslant k} \int_{S \Omega}\left|D^{\alpha} u(x)\right|^{p} d_{M}^{k-(k-|\alpha|) p}(x) d x\right)^{1 / p}<\infty
\end{aligned}
$$

( $R>0$ is a sufficiently large number). This result can be extended to more general domains $\Omega$ and sets $M$. Define $H_{(j, p, M}^{k, p}\left(\Omega ; d_{M}, \varepsilon\right)$ as the closure of $C_{M}^{\infty}(\bar{\Omega})$ in $H_{(j)}^{k, p}\left(\Omega ; d_{M}, \varepsilon\right)$.

TheOREM 2. Let $p, k, \varepsilon, \Omega$ and $M$ satisfy the assumptions of Theorem 1 and let $j=1, \ldots, k$. Then

$$
H_{(j)}^{k, p}\left(\Omega ; d_{M}, \varepsilon\right) \hookrightarrow H_{(j, M}^{k, p}\left(\Omega ; d_{M}, \varepsilon\right) \hookrightarrow W_{M}^{k, p}\left(\Omega ; d_{M}, \varepsilon\right) .
$$

Theorem 3. Let $p, k, m, \Omega$ and $M$ satisfy the assumptions of Proposition. Let $\varepsilon=j p+m-N$ for some $j=1, \ldots, k$. Then

$$
W_{M}^{k, p}\left(\Omega ; d_{M}, \varepsilon\right) \hookrightarrow H_{(j)}^{k, p}\left(\Omega ; d_{M}, \varepsilon\right) .
$$

Proof of Theorem 2 or 3 can be done step by step as the proofs of Theorem 1 or of Proposition (Theorem 2.3 in [1]) and the corresponding theorem in [2].

\section{Traces}

In this section we suppose that the domain $\Omega$ has a Lipschitzian boundary, i.e. there exist a finite number $m$ of coordinate systems $\left(y_{i}^{\prime}, y_{i N}\right), y_{i}^{\prime}$ $=\left(y_{i 1}, \ldots, y_{i . v_{-1}}\right)$ and of functions $a_{i}=a_{i}\left(y_{i}^{\prime}\right)$ Lipschitzian on the closures of the $(N-1)$-dimensional cubes $2 \Delta_{i}=\left\{y_{i}^{\prime} ;\left|y_{i j}\right|<2 \delta\right.$ for $j=1, \ldots, N-1$; $(i=1, \ldots, m)$ and such that:

(i) for each $x \in \partial \Omega$ there is at least one $i \in\{1, \ldots, m\}$ such that $x$ $=\left(y_{i}^{\prime}, y_{i N}\right)$ and $y_{i N}=a_{i}\left(y_{i}^{\prime}\right), y_{i}^{\prime} \in \Delta_{i}=\left\{y_{i}^{\prime} ;\left|y_{i j}\right|<\delta\right.$ for $\left.j=1, \ldots, N-1\right\}$,

(ii) there exists $\beta>0$ such that the sets $B_{i}=\left\{\left(y_{i}^{\prime}, y_{i N}\right) ; y_{i}^{\prime} \in 2 \Delta_{i}, a_{i}\left(y_{i}^{\prime}\right)\right.$ $-2 \beta<y_{i N}<a_{i}\left(y_{i}^{\prime}\right)+2 \beta$; satisfy

and

$$
U_{i}=B_{i} \cap \Omega=\left\{\left(y_{i}^{\prime}, y_{i N}\right) ; y_{i}^{\prime} \in 2 \Delta_{i}, a_{i}\left(y_{i}^{\prime}\right)-2 \beta<y_{i N}<a_{i}\left(y_{i}^{\prime}\right)\right\}
$$

$$
\Gamma_{i}=B_{i} \cap \partial \Omega=\left\{\left(y_{i}^{\prime}, y_{i N}\right) ; y_{i}^{\prime} \in 2 \Delta_{i}, y_{i N}=a_{i}\left(y_{i}^{\prime}\right)\right\}
$$

$(i=1, \ldots, m)$.

Further, let us suppose that the set $M$ is a subset of the boundary $\partial \Omega$. It is easily seen that conditions (a), (b), (c) on $\Omega$ and $M$ from Section 2 are 
satisfied. Set $M_{i}=M \cap B_{i}$. By. $L^{p}\left(\partial \Omega ; d_{M}, \varepsilon\right)$ we denote the space of functions $u$ defined a.e. on $\partial \Omega$ and such that the surface integral

$$
\left(\int_{\delta \Omega}|u(x)|^{p} \cdot d_{M}^{e}(x) d S(x)\right)^{1 / p}
$$

is finite.

It can be proved that

$$
\left[\sum_{i=1}^{m} \int_{\Delta_{i}}\left|u\left(x_{i}^{\prime}, a_{i}\left(x_{i}^{\prime}\right)\right)\right|^{p} d_{M_{i}}^{z}\left(x_{i}^{\prime}, a_{i}\left(x_{i}^{\prime}\right)\right)\right]^{1 / p},
$$

where we put $d_{\phi}(x)=1$, is a norm in $L^{p}\left(\partial \Omega ; d_{M}, \varepsilon\right)$ which is equivalent to the norm (15).

We shall study the existence of traces on $\Gamma_{i}$ of functions from $H^{k, p}\left(\Omega ; d_{M}, \varepsilon\right)$ for some index $i=1, \ldots, m$. If $M_{i}=\emptyset$, then

$$
0<\min (\beta, \delta) \leqslant d_{M_{i}}(x)<\operatorname{diam} \Omega \text { for } x \in B_{i}
$$

and the problem can be reduced to the non-weighted case which is well known.

Thus, suppose $M_{i} \neq \varnothing$. We shall omit the index $i$. Take $x^{\prime} \in \Delta$ and suppose first that $\left(x^{\prime}, a\left(x^{\prime}\right)\right) \notin M$, i.e. $d_{M}\left(x^{\prime}, a\left(x^{\prime}\right)\right)>0$. Following the considerations in the proof of Theorem 2.6 in [3] we can write for $u \in C^{\infty}(\bar{\Omega})$ and for

$$
a\left(x^{\prime}\right)-\min \left(\beta, d\left(x^{\prime}, a\left(x^{\prime}\right)\right)\right)<s<a\left(x^{\prime}\right)
$$

that

$$
\begin{aligned}
\left|u\left(x^{\prime}, a\left(x^{\prime}\right)\right)\right|^{p} & \leqslant 2^{p-1}\left\{\left|u\left(x^{\prime}, s\right)\right|^{p}+\left(\int_{s}^{a\left(x^{\prime}\right)}\left|D_{N} u\left(x^{\prime}, t\right)\right| d t\right)^{p}\right\} \\
& \leqslant 2^{p-1}\left\{\left|u\left(x^{\prime}, s\right)\right|^{p}+d_{M}\left(x^{\prime}, a\left(x^{\prime}\right)\right)^{p-1}\right. \\
& \left.\times \int_{a\left(x^{\prime}\right)-d_{M}\left(x^{\prime}, a\left(x^{\prime}\right)\right)}^{a\left(x^{\prime}\right)}\left|D_{N} u\left(x^{\prime}, t\right)\right|^{p} d t\right\} .
\end{aligned}
$$

The triangle inequality and the Lipschitz property of the function $a$ yield

$$
\frac{1}{2} \leqslant \frac{d_{M}\left(x^{\prime}, a\left(x^{\prime}\right)\right)}{d_{M}\left(x^{\prime}, s\right)} \leqslant c_{1}
$$

for $s$ satisfying (17).

Integrating (18) with respect to $s$ from $a\left(x^{\prime}\right)-d_{M}\left(x^{\prime}, a\left(x^{\prime}\right)\right)$ to $a\left(x^{\prime}\right)$, using estimates (19) and integrating over $\Delta^{*}=\left\{x^{\prime} \in \Delta ;\left(x^{\prime}, a\left(x^{\prime}\right)\right) \notin M\right\}$ we obtain

$$
\begin{aligned}
& \int_{\Delta^{*}}\left|u\left(x^{\prime}, a\left(x^{\prime}\right)\right)\right|^{p} d_{M}^{e-p+1}\left(x^{\prime}, a\left(x^{\prime}\right)\right) d x^{\prime} \\
& \quad \leqslant c_{2}\left\{\int_{U}|u(x)|^{p} d_{M}^{\varepsilon-p}(x) d x+\int_{U}\left|D_{N} u(x)\right|^{p} d_{M}^{\varepsilon}(x) d x\right\} .
\end{aligned}
$$

The last estimate together with Theorem 1 implies 
TIIIORFN 4. I 't I $p, a \in \mathbb{R}$. Then there exists a unique hounded linear operator $Z: H^{1, p}\left(\Omega ; d_{M}, \varepsilon\right) \rightarrow L^{p}\left(\partial \Omega-M ; d_{M}, \varepsilon-p+1\right)$ such that $Z_{u}$ $=\left.u\right|_{\partial \Omega-M} f\left(o r\right.$ all $\| \in C_{M}^{\infty}(\bar{\Omega})$.

By the salme method one can prove

TIII ORI:4 5. I "1 $1, p . x,: \in R$. Then there exists a unicue hounded linear operator $Z$ from $H_{(i)}^{1} p\left(\Omega ; d_{M}, \varepsilon\right)$ into the Lebesgue space $L^{p}$ on $\partial \Omega-M$ will lhe weighth $d_{M}^{n-1+1}(x)\left(\log \frac{R}{d_{M}(x)}\right)$.

Now, we turn our attention to the case $\left(x^{\prime}, a\left(x^{\prime}\right)\right) \in M$. Simple examples show that functions from $H^{k . p}\left(\Omega ; d_{M}, \varepsilon\right)$ for $: \cdots p-1$ may have singularities on 11 - although there is a dense set of functions vanishing near $M$. On the wher hand, if $: \because p-1$ and $u \in H^{k, p}\left(\Omega: d_{M},(x)\right.$, then

$$
\int_{u\left(x^{\prime}\right)-\beta}^{a\left(x^{\prime}\right)}\left|u\left(x^{\prime}, s\right)\right|^{p} d_{M}^{\varepsilon-p}\left(x^{\prime}, s\right) d s+\int_{u\left(x^{\prime}\right)-\beta}^{a\left(x^{\prime}\right)}\left|D_{N} u\left(x^{\prime}, s\right)\right|^{p} d_{M}^{\varepsilon}\left(x^{\prime}, s\right) d s<\propto
$$

for a.e. $x^{\prime} \in \Delta$. By the Hölder inequality we have for $a\left(x^{\prime}\right)-\beta \cdot s \cdot s+h$ $a\left(x^{\prime}\right)$

$$
\begin{aligned}
\mid u\left(. x^{\prime}, s+h\right) & -u\left(x^{\prime}, s\right) \mid \\
& \leqslant\left(\int_{s}^{s+h}\left|D_{N} u\left(x^{\prime}, t\right)\right|^{p} d_{M}^{e}\left(x^{\prime}, t\right) d t\right)^{1 / p}\left(\int_{s}^{s+h} d_{M}^{-\varepsilon /(p-1)}\left(x^{\prime}, t\right) d t\right)^{(p-1) / p}
\end{aligned}
$$

where the first term on the right-hand side is bounded for a.e. $x^{\prime}$ and the second is $o(1)$ as $h \rightarrow 0$. Hence, the function $u$ is uniformly continuous on almost all lines $x^{\prime}=$ const and there exists a finite limit

$$
\lim _{t \cdot u\left(x^{\prime}\right)} u\left(x^{\prime}, t\right)=g\left(x^{\prime}\right)
$$

which must be zero because of the convergence of the first integral in (21). Unfortunately, such considerations do not work if $::=p-1$. Nevertheless, we have

I Fum. Let $0<a<h<1,0<x<\beta<x$ and $1<p<x$. Then for each finction $u \in H^{1 . p}\left((0,1) ; d_{: 0}, p-1\right)$ such that $u(a)=\alpha, u(b)=\beta$,

$$
\int_{a}^{b}|u(x)|^{p} \frac{d x}{x}+\int_{a}^{b}\left|u^{\prime}(x)\right|^{p} x^{p-1} d x \geqslant 2^{1-p} \frac{(p-1)^{1 / p}}{p}|u(b)-u(a)|^{p} .
$$

Proof. We shall only give a sketch of a rather technical proof. The Euler equation of the convex functional

$$
J(u)=\prod_{a}^{b}|u(x)|^{p} \frac{d x}{x}+\prod_{a}^{b}\left|u^{\prime}(x)\right|^{p} \cdot x^{p-1} d x
$$


has a general solution $u_{0}(x)=A \cdot x^{j}+B x^{-i}$, where $\lambda=(p-1)^{-1 / p}$. If we insert $u_{0}$ in $J$ taking into account the values $u_{0}(a)=\alpha, u_{0}(b)=\beta$, we can estimate $J\left(u_{1},\right)=\min J(u)$ from below by the right-liand side of (23).

Now, suppose that the limit (22) does not exist. Then we can choose an oscillating sequence of values $u\left(a_{n}\right)$ such that $a_{n}{ }^{\prime} u\left(x^{\prime}\right)$ and applying the lemma on the intervals $\left(a_{2 n-1}, a_{2 n}\right)$ we get a contradiction with (21). Hence. the limit (22) exists and must be finite for a.e. $x^{\prime}$ because of (21).

In this way we have proved

THEOREY 6. If $\varepsilon \leqslant p-1$, then functions from $H^{k . p}\left(\Omega ; d_{M}, \varepsilon\right)$ have zero races on $M$.

Remark 2. The results of this section can be easily reformulated for the spaces $W^{k \cdot p}\left(\Omega ; d_{M}, \varepsilon\right)$ and $W_{M}^{k . p}\left(\Omega ; d_{M}, \varepsilon\right)$, if we use Theorems $1,2,3$ and Proposition.

Remark 3. We treated the question of existence of traces only. The problem of full characterization of traces by direct and inverse theorems is still open. For certain results with $M=\partial \Omega$ we refer e.g. to [4], [6].

\title{
References
}

[1] D. E. Edmunds, A. Kufner and J. Rákosnik, Embeddings of Soboler spaces with weights if porier type. Z. Anal. Anwendungen 4 (1) (1985). 25 .34.

[2] J. Kadlec and A. Kufner, Characterization of functions with zero traces by integrals with weight functions I, II, Casopis Pěst. Mat. 91 (1966), 463-471; 92 (1967). 16-28.

[3] A. K ufner, Einige Eigenschaften der Sobolevschen Räume mit Belegungsfinktion, Czechoslovak Math. J. 15 (90) (1965), 597-620.

[4] S. M. Nikol'skii, Approximation of functions of seceral cariables and embedding theorems, 2nd ed., Nauka, Moscow 1977 (Russian).

[5] J. Rak osnik, On imbeddings of Sobolet spaces with power-lype weights. Proc. Conference on Approximation Theory, Kiev 1983 (to appear).

[6] B. V. Tandit, On traces of functions from the class $W_{\text {pap }}^{r}$, Differentsialnye Uravnicnia 16 (198()). 2062 2074.

[7]- H. Triebel, A remark on the paper by D. E. Edmunds, A. Kufiner and J. Raikosnik "Embeddings of Sobolev spaces with weights of power type", Z. Anal. Anwendungen 4 (1) (1985), 3538.

\author{
Presented to the Semester \\ Approximation and Function Spaces \\ February 27-Ma! 27, 1986
}

